\title{
Estudo de Nanoantenas Patch de Grafeno na Faixa de Terahertz
}

\author{
Gustavo L. Silvano e Karlo Q. da Costa
}

\begin{abstract}
Resumo-A demanda para se alcançar faixas de frequências cada vez maiores acabam por produzir diversas pesquisas. De fato, pesquisadores recentes conseguiram isolar folhas de grafeno de modo que características únicas começaram a ser observadas. Muitas aplicações podem ser obtidas com a utilização de grafeno, entre elas temos a construção de nanoestruturas ressonantes na faixa de THz. Esse artigo irá estudar a seção reta de absorção de quatro estruturas de grafeno, variando sua dimensão, com o objetivo de estudar suas ressonâncias.
\end{abstract}

\section{Palavras-Chave-Grafeno, $\mathrm{THz}$, nanoantenas.}

Abstract-The demand to reach frequency bands even greater is producing lots of researches. In fact, researchers recently succeeded in isolating a sheet of graphene, in a way that unique characteristics started to be observed. Many applications can be achieved with the utilization of graphene, between them we have the construction of nanostructures resonant in the $\mathbf{T H z}$ band. This article will study the cross-section absorption of four structures of graphene, ranging its dimension, with the objective of study its resonance.

Keywords—Graphene, $\mathrm{THz}$, nanoantennas.

\section{INTRODUÇÃO}

Estruturas compostas de grafeno vêm sendo alvo de várias pesquisas pelo mundo. De fato, as possibilidades provenientes de tal material são bem promissoras e irão alterar várias áreas, como a física, uma vez que diversos fenômenos físicos podem ser melhores estudados graças ao grafeno, e a eletrônica, onde diversas aplicações, como transistores de altíssima velocidade que prometem levar a velocidade de processamento para a faixa de $\mathrm{THz}$ [1] e por fim a geração de energia, onde placas solares transparentes de alta eficiência podem ser concebidas [2].

O grafeno nada mais é do que uma "folha" de átomos de carbono, de duas dimensões, com a espessura de um átomo, ou seja, eles se encontram dispostos lado a lado, em uma distribuição hexagonal, não havendo empilhamento. De fato, uma folha completamente isolada de grafeno é algo muito difícil de ser encontrado, porém novas técnicas convergem para tal.

Nesse artigo, antenas de grafeno serão estudadas, onde se terão quatro estruturas básicas: em forma de retângulo, de elipse, de círculo e de triângulo. Será calculada a seção reta de absorção $\left(\sigma_{\text {abs }}\right)$ para cada estrutura através de uma análise paramétrica onde se irá variar o comprimento $\mathrm{L}$, com a finalidade de se observar a variação da seção reta de absorção de modo a sintonizar a ressonância da estrutura de acordo com sua dimensão. Em seguida será analisada a distribuição de corrente de cada estrutura, através de um gráfico bidimensional, onde foi utilizada a frequência de ressonância.

\section{DESENVOLVIMENTO TEÓRICO}

As dimensões das estruturas construídas estão presentes na Fig. 1. Pode-se perceber que a dimensão L é constante para todas as estruturas e ela irá variar em $5,3,1$ e $0.5 \mu \mathrm{m}$, sendo que para as antenas retangulares e elípticas não houve convergência para a simulação de $0,5 \mu \mathrm{m}$ devido a incapacidade computacional disponível.

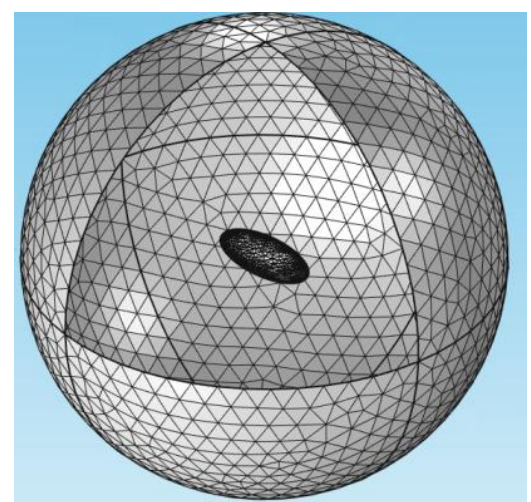

Fig. 1. Geometrias triangulares, circulares, retangulares e elípticas das nanoantenas de grafeno analisadas. As antenas são irradiadas por uma onda plana polarizada ao longo da dimensão L com incidência normal.

A variação da dimensão $\mathrm{W}$ irá acompanhar diretamente a variação de $\mathrm{L}$, de modo que, para a simulação da estrutura retangular e elíptica, obedecerá a proporção de $2 \cdot \mathrm{L}$.

O grafeno é modelado nas simulações através de sua condutividade. Porém como estamos falando de uma estrutura de duas dimensões, tal característica passa a ser anisotrópica, variando somente nos eixos $\mathrm{X}$ e $\mathrm{Y}$, e em $\mathrm{Z}$ é nula. A equação da condutividade foi retirada de [3]

Para as simulações realizadas será utilizado o software COMSOL 4.2a. A Fig. 2 mostra a implementação das estruturas no programa, onde se tem presente também a malha de cada estrutura.

O ambiente onde as antenas se encontram é definido por uma esfera de ar com raio de $4 \cdot \mathrm{L}$, onde foi aplicada a condição de espalhamento nas fronteiras. Como o COMSOL se baseia no método dos elementos finitos, se torna necessário a criação de uma malha. $\mathrm{O}$ valor médio da quantidade de elementos em cada malha é de 400.000, sendo esse o máximo obtido pelas máquinas utilizadas. Com isso, as simulações variavam de $1 \mathrm{~h}$ e $30 \mathrm{~min}$ a $2 \mathrm{~h}$.

Gustavo L. Silvano e Karlo Q. da Costa, Laboratório de Eletromagnetismo, Universidade Federal do Pará, Tucuruí-PA, Brasil, E-mails: gustavolsilvano@gmail.com, karlo@ufpa.br. Este trabalho foi parcialmente financiado pelo $\mathrm{CNPq}$. 

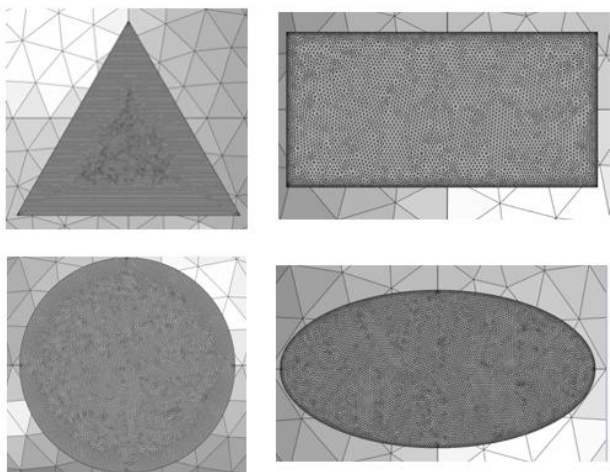

Fig. 2. Implementação no software COMSOL 4.2a das geometrias propostas.

\section{RESULTADOS}

O resultado das simulações é a seção reta de absorção, mostradas na Fig. 3 e 4 . Além disso, se tem presente a distribuição de corrente na ressonância para todas as estruturas onde $\mathrm{L}=5 \mu \mathrm{m}$.

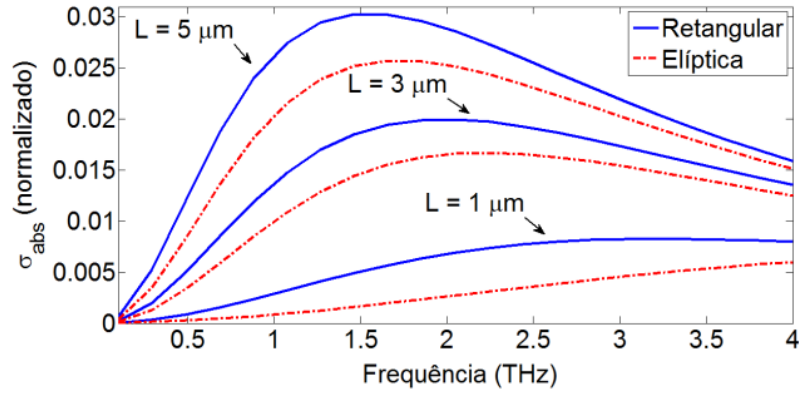

Fig. 3. Seção reta de absorção obtido para a antena retangular e elíptica, onde o $\mathrm{L}$ foi variado em 5,3 e $1 \mu \mathrm{m}$, sendo $\mathrm{W}=2 \cdot \mathrm{L}$. As linhas azuis solidas são obtidas através de um programa desenvolvido no Matlab utilizando o

MoM, já as circunferências vermelhas são obtidas pelo COMSOL

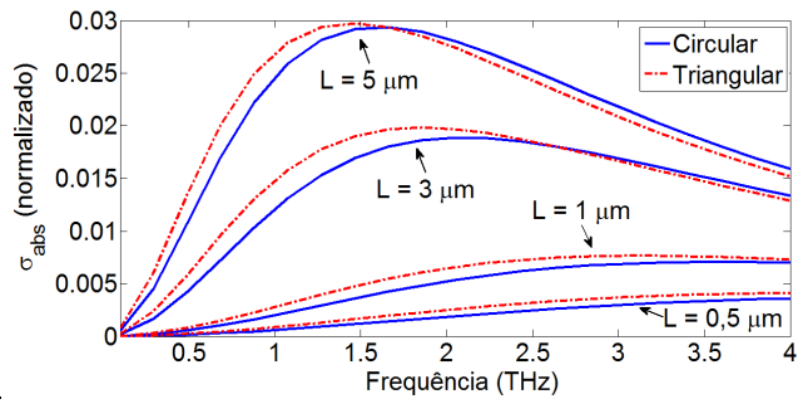

Fig. 4. Seção reta de absorção obtido para a antena triangular e circular, onde o $\mathrm{L}$ foi variado em $5,3,1$ e $0,5 \mu \mathrm{m}$. As linhas azuis solidas são obtida através de um programa desenvolvido no Matlab utilizando o MoM, já as circunferências vermelhas são obtidas pelo COMSOL.

Com os resultados obtidos, percebe-se que a variação do $\sigma_{a b s}$ varia bastante com a mudança da dimensão. De fato, observou-se que as estruturas menores apresentavam uma redução do seu valor de $\sigma_{\mathrm{abs}}$, além de uma mudança da frequência de ressonância para valores maiores.

$\mathrm{Na}$ Fig. 5 é mostrado a corrente induzida para as frequências de ressonância de cada estrutura. Pode-se perceber que o valor da frequência é bastante próximo entre as estruturas, o que está certo uma vez que elas possuem a mesma dimensão.
A Tabela I mostra uma comparação entre os valores de ressonância obtido para cada estrutura onde se tem presente os valores calculados pelo COMSOL e pelo MoM [4].

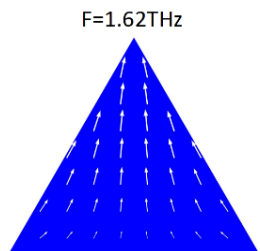

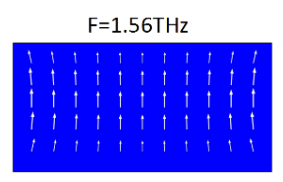

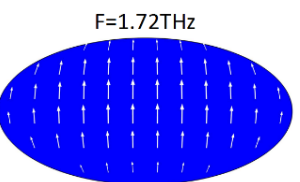

$\mathrm{F}=1.44 \mathrm{THz}$

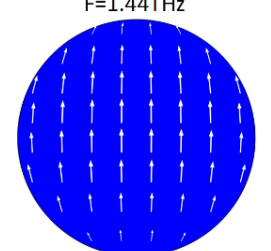

Fig. 5. Geometrias triangulares, circulares, retangulares e elípticas das nanoantenas de grafeno analisadas. As antenas são irradiadas por uma onda plana polarizada ao longo da dimensão L com incidência normal.

TABELA I. VALORES DE RESSONÂNCIA OBTIDOS PARA AS QUATRO GEOMETRIAS E PARA CADA VALOR DE L. ESTÃO PRESENTES RESULTADOS OBTIDOS ATRAVÉS DO COMSOL E DO MOM [4].

\begin{tabular}{|c|l|c|c|c|c|}
\cline { 3 - 6 } \multicolumn{2}{|c}{} & Triangular & Circular & Elíptica & Retangular \\
\hline $\begin{array}{c}5 \\
{[\mu \mathrm{m}]}\end{array}$ & COMSOL & 1,46 & 1,66 & 1,66 & 1,46 \\
\cline { 2 - 6 } & MoM & 1,39 & 1,59 & 1,71 & 1,55 \\
\hline $\begin{array}{c}3 \\
{[\mu \mathrm{m}]}\end{array}$ & COMSOL & 1,85 & 2,05 & 2,19 & 2,05 \\
\cline { 2 - 6 } & MoM & 1,87 & 2,03 & 2,24 & 2,03 \\
\hline $\begin{array}{c}1 \\
{[\mu \mathrm{m}]}\end{array}$ & COMSOL & 3,22 & 3,61 & 3,9 & 3,22 \\
\cline { 2 - 6 } & MoM & 3,16 & 3,64 & 4 & 3,47 \\
\hline $\begin{array}{c}0,5 \\
{[\mu \mathrm{m}]}\end{array}$ & COMSOL & 4 & 4 & - & - \\
\cline { 2 - 6 } & MoM & 4 & 4 & - & - \\
\hline
\end{tabular}

\section{CONCLUSÕES}

Como os resultados obtidos para as quatro geometrias, percebe-se uma semelhança entre nanoantenas e antenas de microondas. Isso porque o parâmetro de seção reta de absorção se mostrou menor para geometrias com bordas arredondadas e menores. Além disso observou-se que antenas de diferentes formatos mas com dimensão $\mathrm{L}$ iguais possuíam uma ressonância próxima entre si. Com a simulação paramétrica, se mapeou o comportamento da ressonância, que pode ser utilizado para sintonizar a nanoantena para ressoar na frequência desejada.

\section{REFERÊNCIAS}

[1] F. Schwierz, "Graphene transistors," Nature Nanotechnology, vol. 5, pp. 487-496, 2010.

[2] X. Wang, L. Zhi, and K. Mu llen, Transparent, "Conductive graphene electrodes for dye-sensitized solar cells," Nano Letters, vol. 8, pp. 323$327,2008$.

[3] I. Llatser, C. Kremers, A. Cabellos-Aparicio, J. M. Jornet, E. Alarco, D. N. Chigrin, "Graphene-based nano-patch antenna for terahertz radiation," Photonics and Nanostructures - Fundamentals and Applications, in press.

[4] K. Q. Costa, et al, "Scattering Analysis of Graphene Nanoantennas with Different Shapes, ” IX International Conference on Wireless and Mobile Communications, a ser aprovado. 